\title{
Model-Driven Service Level Management ${ }^{*}$
}

\author{
Anacleto Correia ${ }^{1,2}$ and Fernando Brito e Abreu ${ }^{1}$ \\ ${ }^{1}$ QUASAR/CITI/Faculdade de Ciências e Tecnologia/Universidade Nova Lisboa, Caparica \\ ${ }^{2}$ Escola Superior de Tecnologia/Instituto Politécnico de Setúbal, Setúbal \\ accorreialfct.unl.pt, fba@di.fct.unl.pt
}

\begin{abstract}
Service-level agreements (SLA) definition and monitoring are open issues within the IT Service Management (ITSM) domain. Our main goals are to propose a model-based approach to IT services SLA specification and compliance verification. The specification will be accomplished by proposing a SLA language - a domain specific language for defining quality attributes as non functional requirements (NFRs) in the context of ITSM. This will allow that SLA monitoring and compliance validation at a level of abstraction that is understood by the stakeholders involved in the service specification.
\end{abstract}

Keywords: IT Service Management, ITIL, SLA, MDA; DSL, NFR, BPMN.

\section{Introduction}

Most organizations rely on Information Technology (IT) services to support their business processes. IT services are built upon the technical infrastructure, systems and application software. The set of processes that allow planning, organizing, directing and controlling the provisioning of IT services is called an IT Service Management (ITSM). Several ITSM frameworks have been proposed, such as the ITIL [1]. In ITIL, one of the most relevant processes is service level management. In order to support the IT services, IT providers use two kinds of applications: service management applications, which allow tracking IT services incidents and problems; and systems management tools, for monitoring and controlling networks, systems or applications.

Among the concerns within the service level management process, are for instance, the requirements for services availability, performance, accuracy, which are specified in terms of service-level agreements (SLA). In a SLA, such requirements, known as quality attributes, are quantitatively bounded (e.g. maximum time to recover). However, although those requirements are non-functional in nature, and many nonfunctional requirements techniques have been proposed in the literature [2], those techniques are not being used in SLA specification.

SLA definition and monitoring are identified as open issues within the ITSM domain [3]. In this research plan we are going to focus our attention on three specific problems in this context: The first problem arises from the current practice in SLA specification for IT services, mostly based in templates filled with natural language

\footnotetext{
* The work presented herein was partly supported by the VALSE project of the CITI research center within the Department of Informatics at FCT/UNL in Portugal.
} 
descriptions, and results not amenable to the automation of SLA compliance verification. Since SLA definition are subjective in nature it renders the second problem: it is not clear to all involved stakeholders which are the activities upon which SLA compliance should be verified, and how their non-compliance will affect the evolution in the process describing the IT service delivery. The third problem concerns a semantic gap which occurs in SLA compliance verification. This gap comes from the fact that compliance checking has been relying mostly in Quality of Service (QoS) information from systems management applications, instead of consolidated data presented at the process model representation of the IT service.

In the rest of this paper we will present a survey on the current state of the art of SLAs (section 2) and our proposal to deal with SLAs in the ITSM domain (section 3).

\section{Related Work}

When discussing related work, it is useful to have a common taxonomy that fosters a more systematic study of different approaches and also helps identifying the issues, which are not overcome by current approaches, and our proposal intends to tackle. Our taxonomy for describing the entries of the survey of the current state of the art in SLA specification and compliance validation (see Table 1) is based in the following dimensions: Domain: it refers to the technological context at which the SLA is defined and evaluated. (1) ITSM - regarding processes of an ITSM framework; (2) SOA - concerning web services interacting in an SOA environment; (3) System Management - related with services of IT infrastructure assessed by QoS parameters. Formalization: it refers to the level of formalism used for SLA specification. (1) Formal - mathematically-based techniques amenable of rigorous proof; (2) Semi-formal although the representation might not be a complete description of reality, the representation itself and the system description are equivalent; (3) Informal - descriptions in natural language usually using a structured template form. Abstraction Level: it refers to the kind of representation used in SLA. (1) Model-based - diagrammatic models for SLA elicitation and specification; (2) Hybrid - a blended approach using a diagrammatic notation supplemented by a textual language; (3) Textual - specification exclusively based in textual language. Verification Compliance: it refers to the level at which the SLA validation occurs. (1) Model-based - the SLA compliance is checked at the level of models; (2) Message-based - the compliance verification is based in the exchanged messages; (3) Data-based - the compliance verification is based on information stored in databases or the like repositories, by applications that deal with and support special types of IT infrastructure or specific IT processes; (4) Absent - there is no SLA compliance checking.

The most well-known approaches on SLAs are related with SOA (Web Service Level Agreement (WSLA) [4], the Web Services Agreement Specification (WSAgreement) specification [5], Web Services Offering Language (WSOL) [6], the Web Service Management Layer (WSML) [7] and the Web Service Modeling Ontology (WSMO) [8], Rule-Based Service Level Agreements (RBSLA) [9] and the SLAng [10]). These approaches only address computational processes and are not able to deal with processes where humans are involved such the ones covered of ITSM. Moreover 
the verification of compliance is made at an implementation instead of at a model level most suitable for common stakeholders.

There are also available, a number of service management applications that support the ITSM service level management process [11], as well as systems management tools [12], that monitor network, IT devices and applications at QoS parameters level. Albeit the former applications are related with the ITSM domain, they have issues, as we highlighted in section 1, such as the informal specification of SLA and the monitoring and compliance verification, based mostly in scattered and not integrated repositories of data. The latter applications have also these kinds of issues, and moreover their domain is mainly the technical infrastructure.

Table 1. Summary of current SLA approaches to specification and validation

\begin{tabular}{lcccc}
\hline Approach & Domain & Formalization & $\begin{array}{l}\text { Abstraction } \\
\text { Level }\end{array}$ & $\begin{array}{l}\text { Verification } \\
\text { Compliance }\end{array}$ \\
\hline$[4][5][6][7][8][9]$ & $(2)$ & $(1)$ & $(3)$ & $(2)$ \\
{$[10]$} & $(2)$ & $(1)$ & $(2)$ & $(2)$ \\
{$[11]$} & $(1)$ & $(3)$ & $(3)$ & $(3)$ \\
{$[12]$} & $(3)$ & $(3)$ & $(3)$ & $(3)$ \\
\hline
\end{tabular}

\section{SLA Specification and Validation Environment}

Our proposal for the specification and validation of SLA compliance of IT services is supported by an environment, the SLA specification and validation Environment (SLAEnv). The SLAEnv architecture addresses two main phases in the SLA life cycle: the SLA specification phase, when a negotiation process takes place among the stakeholders; and the validation phase, which includes the monitoring, reporting, evaluating and improving of the SLA.

The negotiation step aims to define the conditions of the SLA contract. An SLA Editor supports this activity. The SLA Editor must be built based in the Process Metamodel and SLA Metamodel, using a DSL Tool. For the Process Metamodel definition the foundation is the BPMN 2.0 specification. On the other hand the SLA metamodel specification is based in techniques of non functional requirements (NFRs) elicitation. The output of the SLA Editor is an SLA Contract expressed in a SLA language, compliant with the SLA metamodel, and additional integrity constraints expressions written in the OCL.

The conditions of an SLA contract are the input for an SLA Evaluator. System states (snapshots of a running system) could be created and manipulated during an evaluation. Information about a system state will be attained by querying the Process Modeler Animator for IT service execution. For each snapshot OCL constraints can be checked. By evaluating OCL expressions we could gather detailed information about the system's SLA conformance. Graphical views (eg. dashboard or balanced scorecard) of system's state can be provided to the SLA Visualizer.

The Process Modeler Animator is a BPMN console where IT services are depicted and animated as BPMN process diagrams. An IT service specification, can be persisted in a standard format (e.g. XMI), which will be the Process Model repository. 
This definition is required for the SLA contract definition, since it will allow that hooks, concerning SLA quality attributes, could be associated to BPMN diagram elements (activities, gateways, etc.). Those hooks will allow the SLA Evaluator to trace an IT service instance execution to the corresponding SLA contract quality attributes thresholds. Therefore, the SLA Evaluator is the corner stone of the environment since it joins the specification and validation phases of an SLA. This is done by matching the SLA contract assertions with the IT service data execution, and making available the resulting snapshot to the visualization component.

This approach, unlike the mentioned in section 2, is tailored for SLA monitoring and compliance verification in the ITSM domain. Moreover, it allows a semi-formal, and model based specification and conformance checking of SLA. The validation process of this proposal will take place in an IT service management project on the domain of financial self-service systems.

\section{Conclusions}

Since SLA definition and monitoring are open issues within the ITSM domain, this research work intends to address it by proposing a model-based approach to IT services SLA specification and compliance verification. The specification part will be accomplished by proposing a SLA language. This language will be derived by way of a domain specific language that is intended to be able to specify the quality attributes, also known as non functional requirements, in the context of ITSM. The main contribution of this proposal is to allow SLA compliance verification to be made at the same abstraction level of specification, i.e., at a model level, filling the semantic gap between stakeholders.

\section{References}

1. ITIL3, OGC. Summary, ITIL Version 3: ITSMF - ITSM Forum (2007)

2. Matoussi, A., Laleau, R.: A Survey of NFR in Soft. Dev. Process. DI UP 12 (2008)

3. Coyle, D.M., Brittain, K.: Magic Quadrant for IT Service Desk. In: Gartner 2009 (2009)

4. IBM. WSLA Language Specification, version 1.0. (2001)

5. Andrieux, A., Czajkowski, K., et al.: WS-Agreement. Open Grid Forum (2007)

6. Tosic, V., Lutfiyya, H., Tang, Y.: WSOL. In: AICT-ICIW 2006, p. 156 (2006)

7. Verheecke, B.: Web Services Management Layer, WSML (2003)

8. W3C. Web Service Modeling Ontology, WSMO (2005)

9. Paschke, A.: RBSLA. In: Proceedings of ICCIMCA 2005, vol. 02, pp. 308-314 (2005)

10. Skene, J., Lamanna, D., Emmerich, W.: Precise SLA. In: ICSE 2004, Edinburgh (2004)

11. PinkElephant. PinkVERIFY 3.0 Toolsets (2010)

12. Gläßer, L.: Development and Operation of Application Solutions. Siemens (2005) 